\title{
Checklist de Plecoptera (Insecta) do Estado do Mato Grosso do Sul, Brasil
}

\author{
Lucas Silveira Lecci' \& Karina Ocampo Righi-Cavallaro²
}

1. Faculdades do Vale do Juruena - AJES, Juína, Mato Grosso, Brasil. (Iucaslecci@gmail.com)

2. Universidade Federal de Mato Grosso do Sul, Centro de Ciências Exatas e Tecnologia, FAENG, Campo Grande, MS. (karina.righi@gmail.com)

Recebido 30 novembro 2016

Aceito 6 fevereiro 2017

DOI: $10.1590 / 1678-4766 e 2017118$

ABSTRACT. Checklist of Plecoptera (Insecta) from Mato Grosso do Sul State, Brazil. We present here a list of species of the Order Plecoptera found in Mato Grosso do Sul State based on the current literature up to March 2015. One family (Perlidae), one genus (Anacroneuria) and 10 species are recorded.

KEYWORDS. Neotropical region; Anacroneuria; Biota-MS Program.

RESUMO. Apresentamos aqui uma lista das espécies da ordem Plecoptera encontradas no Estado do Mato Grosso do Sul baseados na literatura até março de 2015. Foram registrados uma família (Perlidae), um gênero (Anacroneuria) e 10 espécies.

PALAVRAS-CHAVE. Região Neotropical; Anacroneuria; Programa Biota-MS.

A ordem Plecoptera é relativamente pequena, quando comparada com outras ordens de insetos, por possuir cerca de 3200 espécies descritas (STARK et al., 2009) em 16 famílias, distribuídas por todos os continentes, exceto na Antártida (Zwick, 2000). No Brasil são conhecidas 170 espécies (Froehlich, 2011a, 2012; Bispo \& LeCCI, 2012; AvelinoCAPistrano et al., 2013; BisPo \& Neves, 2014) em duas famílias. A família Gripopterygidae com quatro gêneros pode ser encontrada desde o sul até as regiões montanhosas do Brasil central, e pelo litoral, até o sul e região central do estado da Bahia, já a família Perlidae pode ser encontrada praticamente por todo o país, também apresentando quatro gêneros (FroEHLICH, 2012).

Suas ninfas são aquáticas e podem ser encontradas sob pedras em rios e também em qualquer lugar do rio onde exista oxigênio (GILler \& MALMQVIST, 1998) e alimento. E os adultos, por sua vez, podem ser encontrados sob vegetação ripária ou voando próximo aos corpos d'água nos quais as ninfas são encontradas.

Os imaturos têm uma grande importância ecológica, apresentando um papel relevante na ciclagem de nutrientes e no fluxo de energia dos riachos. Além disso, a ordem Plecoptera juntamente com Ephemeroptera e Trichoptera são indicadores da qualidade da água devido a sua sensibilidade à poluição (ROSENBERG \& RESH, 1993).

\section{MATERIAL E MÉTODOS}

Este checklist foi preparado com base na consulta aos trabalhos de Froehlich $(2007 ; 2010)$ e Righi-CaVAllaro \& LECCI (2010).

\section{RESULTADOS E DISCUSSÃO}

Até os trabalhos de Froehlich (2007) e RighiCavallaro \& Lecci (2010) o estado do Mato Grosso do Sul não possuía nenhuma espécie formalmente descrita ou registrada para a região.

Até o momento, para todo o estado do Mato Grosso do Sul, são encontrados apenas representantes de Perlidae, e somente o gênero Anacroneuria, este com dez espécies, sendo oito descritas pra o estado e duas como registros (Tab. I). Anacroneuria é o gênero dominante na Região Neotropical, sendo que em alguns locais o único a ocorrer (Froehlich, 2002).

Comentários sobre a lista, riqueza do estado comparado com outras regiões. O número de espécies de Plecoptera para o estado do Mato Grosso do Sul é seguramente subestimado, visto a enorme área deste estado que ainda não foi amostrada. Um exemplo disto é que para o estado de São Paulo, com um terço da área, possui 64 espécies registradas (FroeHLICH, 2011b; LeCCI \& FroeHLICH, 2011; BISPO \& LECCI, 2012); isso reflete a deficiência de estudos sobre a ordem Plecoptera para o estado do Mato Grosso do Sul. Os outros estados cuja fauna de plecópteros é melhor conhecida são o Rio de Janeiro e Santa Catarina, com 33 e 23 espécies descritas respectivamente (FroEHLICH, 2011b; Avelino-Capistrano et al., 2013). A plecopterofauna do 
Tab. I. Lista das espécies de Plecoptera do Estado do Mato Grosso do Sul, Brasil.

\begin{tabular}{|c|c|}
\hline Espécie & Distribuição \\
\hline $\begin{array}{l}\text { Anacroneuria atrifrons } \\
\text { Klapálek, } 1922\end{array}$ & Amazonas; Mato Grosso do Sul \\
\hline $\begin{array}{l}\text { Anacroneuria genualis } \\
\text { (Navás, 1932) }\end{array}$ & Mato Grosso do Sul \\
\hline $\begin{array}{l}\text { Anacroneuria melzeri } \\
\text { (Navás, 1932) }\end{array}$ & Mato Grosso do Sul \\
\hline $\begin{array}{l}\text { Anacroneuria pastaza } \\
\text { Stark, } 2001\end{array}$ & Goiás; Mato Grosso do Sul \\
\hline $\begin{array}{l}\text { Anacroneuria ofaye } \\
\text { Froehlich, } 2007\end{array}$ & Mato Grosso do Sul \\
\hline $\begin{array}{l}\text { Anacroneuria guaicuru } \\
\text { Froehlich, } 2007\end{array}$ & Mato Grosso do Sul \\
\hline $\begin{array}{l}\text { Anacroneuria payagua } \\
\text { Froehlich, } 2007\end{array}$ & Mato Grosso do Sul \\
\hline $\begin{array}{l}\text { Anacroneuria otafroehlichi } \\
\text { Righi-Cavallaro \& Lecci, } 2010\end{array}$ & Mato Grosso do Sul \\
\hline $\begin{array}{l}\text { Anacroneuria terere } \\
\text { Righi-Cavallaro \& Lecci, } 2010\end{array}$ & Mato Grosso do Sul \\
\hline $\begin{array}{l}\text { Anacroneuria singularis } \\
\text { Righi-Cavallaro \& Lecci, } 2010\end{array}$ & Mato Grosso do Sul; São Paulo \\
\hline
\end{tabular}

estado do Mato Grosso do Sul, apesar de pouco estudada, está em melhor situação quando comparada a outros estados das Regiões Norte e Nordeste, alguns destes ainda não possuem registro.

Principais grupos de pesquisa e acervos. Em Ribeirão Preto, SP, há o Laboratório de Entomologia Aquática, do Departamento de Biologia da FFCLRP-USP, coordenado pelo Prof. Dr. Claudio Gilberto Froehlich. Em Assis-SP existe o Laboratório de Biologia Aquática, UNESP, coordenado pelo Prof. Dr. Pitágoras da Conceição Bispo. A maior coleção de exemplares do Brasil está no Museu de Zoologia da USP (MZSP), onde se encontra tipos e material determinado de todos os gêneros que ocorrem no país. O Laboratório de Entomologia Aquática, Departamento de Biologia, FFCLRP-USP e o Laboratório de Biologia Aquática, UNESP, campus de Assis, possui uma pequena coleção de referência.

Principais lacunas de conhecimento. No Mato Grosso do Sul, apenas a região de Bonito - mais precisamente a Serra da Bodoquena - foi amostrada, permanecendo assim uma enorme área do estado ainda inexplorada.

Perspectivas de pesquisa para os próximos dez anos. No Brasil há poucos especialistas atuando, porém novos pesquisadores estão sendo formados. Os estudos de cunho ecológico devem aumentar devido à crescente utilidade dos plecópteros em programas de biomonitoramento; neste contexto, estudos taxonômicos devem ser priorizados, o que auxiliará tanto no conhecimento da diversidade local quanto em pesquisas ecológicas.

Agradecimentos. À Fundação de Apoio ao Desenvolvimento do Ensino, Ciências e Tecnologia do Estado de Mato Grosso do Sul (Fundect) e a Superintendência de Ciências e Tecnologia do Estado de Mato Grosso do Sul (Sucitec/MS) pelo convite de participação neste fascículo especial da Biota Neotropica e o suporte financeiro para sua publicação. Os autores são gratos ao Prof. Dr. Claudio Gilberto Froehlich, pelas sugestões e revisão do manuscrito.

\section{REFERÊNCIAS BIBLIOGRÁFICAS}

Avelino-Capistrano, F. S.; De-SouZa, M. R. \& Nessimian, J. L. 2013. Kempnyia puri, a new species of Perlidae (Plecoptera) from Rio de Janeiro, Brazil. Zootaxa 3619(5):554-556.

Bispo, P. C. \& LeCCI, L. S. 2012. Gripopterygidae (Plecoptera) from Paranapiacaba mountains, southeastern Brazil. Annales de Limnologie - International Journal of Limnology 47(4):373-385. (2011)

Froenlich, C. G. 2002. Anacroneuria mainly from southern Brazil and northeastern Argentina (Plecoptera, Perlidae). Proceedings of Biological Society of Washington 115(1):75-107.

Froehlich, C. G. 2007. Three new species of Anacroneuria (Plecoptera: Perlidae) from the State of Mato Grosso do Sul, Brazil. Zootaxa 1461:15-24.

Froenlich, C. G. 2010. Catalogue of Neotropical Plecoptera. Illiesia 6(12):118-205.

Froehlich, C. G. 2012. Plecoptera. In: Rafael, J. A.; Melo, G. A .R.; Carvalho, C. J. B.; Casari, S. A. \& Constantino, R. eds. Insetos do Brasil. Diversidade e Taxonomia. Ribeirão Preto, Holos, p. 257-261.

Froenlich, C. G. 2011a. Kempnyia (Plecoptera) from the Mantiqueira Mountains of Brazil. Zootaxa 2999:20-32.

Froenlich, C. G. 2011b. Checklist of Plecoptera from São Paulo State, Brazil. Biota Neotropica 11(1a): 1-6. Disponível em <http://www. biotaneotropica.org.br/v11 n1a/en/abstract?inventory+bn029110 1a2011>. Acesso em 07.2012.

GILleR, P. S. \& MALMQVist, B. 1998. The Biology of Streams and Rivers. Oxford, Oxford University Press. 296 p.

Lecci, L. S. \& Froenlich, C. G. 2011. Taxonomic revision of Gripopteryx (Pictet, 1841) (Plecoptera: Gripopterygidae). Zootaxa 2792:1-21.

Novaes, M.C. \& Bispo, P.C. 2014. A new species and notes on Perlidae (Plecoptera) from Paraná and Santa Catarina States, southern Brazil. Zootaxa 3765(5):458-468.

Righi-Cavallaro, K. O. \& Lecci, L. S. 2010. Three new species of Anacroneuria (Plecoptera: Perlidae) from Centre-West and Southeast Brazil. Zootaxa 2683:35-44.

Rosenberg, D. M. \& Resh, V. H. 1993. Freshwater monitoring and benthic macroinvertebrates. New York, Chapman \& Hall. 448p.

Stark, B. P.; Froehlich, C. G. \& ZúÑIGa, M. C. 2009. South American Stoneflies (Plecoptera). In: Adis, J.; Arias, J.; Golovatch, S.; WantZen, M. \& Rueda-Delgado, G. Aquatic Biodiversity in Latin America. vol. 5. Moscow, Pensoft Sofia.154 p.

ZwICK, P. 2000. Phylogenetic system and zoogeography of the Plecoptera. Annual Review of Entomology 45:709-746. 